\title{
PREVALÊNCIA DE OBESIDADE E HIPERTENSÃO ARTERIAL EM UMA COMUNIDADE URBANA DO DISTRITO FEDERAL, BRASIL
}

\author{
PREVALENCE OF OBESITY AND HYPERTENSION IN AN URBAN COMMUNITY OF \\ THE FEDERAL DISTRICT, BRAZIL
}

\author{
Maurílio Tiradentes Dutra ${ }^{\mathrm{a}^{*}}$, Karla Gonçalves Martins ${ }^{\mathrm{b}^{*}}$, Diego Batista Reis ${ }^{\mathrm{c}^{*}}$, \\ Débora Kerolayne Santos ${ }^{\mathrm{d}^{\star}}$, Edi Xavier Faria ${ }^{\mathrm{e}^{\star}}$, Paulo César Ramos Araújo ${ }^{\mathrm{f}^{*}}$ \\ mariliotiradentes@gmail.com ${ }^{\mathrm{a}}$, kakazinhacep@gmail.com ${ }^{\mathrm{b}}$, diegobvreis@gmail.com ${ }^{\mathrm{c}}$, deborakarolayned@gmail.com ${ }^{\mathrm{d}}$, \\ edi.xavier6@gmail.com ${ }^{\mathrm{e}}$,prof.pcra@gmail.com ${ }^{\mathrm{f}}$ \\ Escola Técnica de Saúde de Planaltina, Secretaria de Estado de Educação do Distrito Federal, Brasil*
}

\section{RESUMO}

Data de recebimento do artigo: 29/11/2017

Data de aceite do artigo: 17/01/2019

Introdução: A hipertensão arterial e a obesidade configuram graves e cada vez mais emergentes desfechos em saúde pública. Objetivo: o propósito deste estudo foi avaliar a prevalência de sobrepeso, obesidade e hipertensão na comunidade urbana de Planaltina, DF, Brasil. Materiais e Métodos: Participaram do estudo 629 voluntários de 20 a 90 anos de idade que responderam a um questionário de saúde e foram submetidos aos seguintes procedimentos: medida da pressão arterial e avaliação antropométrica. Resultados: A prevalência de hipertensão foi de $31,3 \%$, sendo ligeiramente maior no sexo feminino $(33,0 v s$ 28,1\%) e crescente com o aumento da faixa etária. A prevalência de obesidade foi de $26,6 \%$, sendo similar entre os sexos ( $26,7 \%$ no masculino e $26,5 \%$ no feminino). Excesso de peso (sobrepeso somado à obesidade) foi observado em mais de $50 \%$ da amostra em todas as faixas etárias. Conclusões: As prevalências de sobrepeso, obesidade, excesso de peso e hipertensão foram maiores do que as relatadas pelo inquérito telefônico mais recente do Ministério da Saúde. Esses achados são preocupantes e apontam para a premência de ações do poder público que interfiram positivamente na saúde da população estudada.

Palavras-chave: Obesidade, sobrepeso, pressão arterial, hipertensão

\begin{abstract}
Introduction: Hypertension and obesity are serious and increasingly emerging outcomes in public health. Objective: The aim of this study was to evaluate the prevalence of these chronic diseases in the urban community of Planaltina, DF, Brazil. Materials and Methods: The study included 629 volunteers aged 20 to 90 years who completed a health questionnaire and underwent the following procedures: blood pressure measurements and anthropometric assessment. Results: The prevalence of hypertension was $31.3 \%$, slightly higher in females (33.0 vs $28.1 \%$ ) and increased with increasing age. The prevalence of obesity was $26.6 \%$, which was similar between genders (26.7\% for males and $26.5 \%$ for females). Excess weight (overweight and obesity) was observed in over $50 \%$ of the sample in all age groups. Conclusions: The prevalence of overweight, obesity, excessive weight and hypertension were higher than those reported by the most recent telephone survey of the Ministry of Health of Brazil. These findings are alarming and point to the urgency of government actions that have a positive impact on the health of the population studied.
\end{abstract}

Keywords: Obesity, overweight, blood pressure, hypertension 


\section{Introdução}

A hipertensão arterial (HA) é caracterizada por valores elevados e sustentados de pressão arterial (PA). Trata-se de uma condição crônica, de causa multifatorial, frequentemente associada a alterações funcionais e estruturais de diversos órgãos. A HA está relacionada a alterações metabólicas, com consequente aumento do risco de eventos cardiovasculares fatais e não fatais ${ }^{1}$.

Os fatores de risco para HA incluem idade, gênero, etnia, condição socioeconômica, excesso de peso e outros relacionados ao estilo de vida, como ingestão de sal e sedentarismo ${ }^{1}$. De acordo com o inquérito telefônico do Ministério da Saúde (VIGITEL), a prevalência de HA no Brasil é de $24,8 \% 2$.

A obesidade é uma condição crônica, de etiologia complexa e multifatorial, e resulta da interação de fatores genéticos, emocionais, ambientais e de estilo de vida ${ }^{3}$. Não raro, o excesso de peso relaciona-se com outras condições crônicas, como HA e diabetes ${ }^{4}$. A prevalência de obesidade tem crescido vertiginosamente, alcançando $17,9 \%$ da população das capitais brasileiras em $2014^{2}$.

De fato, o excesso de peso e a HA elevam substancialmente os custos assistenciais em saúde. No entanto, muitos locais ainda carecem de investigação no que diz respeito à prevalência desses desfechos. O conhecimento desses dados é relevante, pois pode nortear ações governamentais e não governamentais de promoção da saúde.

O objetivo do presente estudo foi verificar a prevalência de HA e obesidade na região de Planaltina, Distrito Federal (DF), Brasil.

\section{Metodologia}

O presente estudo é tem caráter transversal. Os dados integram os resultados do estudo "Saúde para Planaltina", conduzido pela Escola Técnica de Saúde de Planaltina (DF). Os procedimentos atendem aos requisitos da Declaração de Helsinki e da resolução 196/96 do Conselho Nacional de Saúde e foram aprovados pelo Comitê de Ética em Pesquisa da Faculdade de Medicina da Universidade de Brasília, sob o número 908.058.
Participaram 629 voluntários $(65,5 \%$ mulheres; $34,5 \%$ homens) com idade entre 20 e 90 anos. A aquisição dos dados ocorreu em diversos locais da comunidade. Os pesquisadores montaram a estrutura necessária para realizar os procedimentos em praças e pontos de encontro comunitário. As pessoas que circulavam pelos locais eram convidadas a participar. Todos os voluntários receberam orientações sobre controle da PA e do índice de massa corporal (IMC).

Após serem informados sobre os procedimentos e darem consentimento esclarecido, todos os voluntários responderam a um questionário de saúde contendo informações sobre diagnóstico de doenças e uso de medicamentos. Para análise da prevalência de HA foi utilizado o diagnóstico que os próprios voluntários informaram no questionário de saúde.

A massa corporal dos participantes foi medida com balança digital portátil com capacidade de $150 \mathrm{~kg}\left(\mathrm{EKS}^{\circ}\right)$. A estatura foi medida utilizando-se estadiômetro portátil (Sanny"). O IMC foi calculado dividindo-se a massa corporal pela estatura ao quadrado $\left(\mathrm{kg} / \mathrm{m}^{2}\right)$ e a classificação de sobrepeso e obesidade obedeceu aos critérios da Associação Brasileira para o Estudo da Obesidade e da Síndrome Metabólica (ABESO) $)^{3}$. Excesso de peso foi definido como $\mathrm{IMC} \geq 25,0 \mathrm{~kg} / \mathrm{m}^{2}$. A análise estatística foi realizada através do pacote estatístico SPSS para Windows (versão 20.0). O teste Qui-Quadrado foi adotado para verificar a associação entre HA, excesso de peso, idade e sexo.

\section{Resultados}

A tabela 1 apresenta a prevalência de obesidade e HA por faixa etária. A prevalência de HA foi de $31,3 \%$ e cresce de acordo com o aumento da faixa etária. Excesso de peso foi observado em $70,3 \%$ do total da amostra, atingindo quase $80 \%$ entre os indivíduos entre 50-59 anos de idade e sendo maior do que $50 \%$ em todas as faixas etárias. 25,4\% da amostra apresentou HA e excesso de peso concomitantemente. 
Tabela 1: Prevalência de sobrepeso, obesidade e HAS por faixa etária $(n=629)$.

\begin{tabular}{lcccccc}
\hline Faixa etária (anos) & N & \% Baixo Peso & \% Peso Normal & \% Sobrepeso & \% Obesidade & \% HA \\
\hline $20-29$ & 90 & 3,3 & 40,0 & 28,9 & 27,8 & 2,5 \\
$30-39$ & 134 & 2,2 & 37,3 & 34,3 & 26,1 & 11,7 \\
$40-49$ & 163 & 0,0 & 24,5 & 46,0 & 29,4 & 19,3 \\
$50-59$ & 131 & 0,0 & 20,6 & 55,0 & 24,4 & 30,5 \\
$\geq 60$ & 111 & 0,9 & 24,3 & 50,5 & 24,3 & 36,0 \\
Total & 629 & 1,1 & 28,6 & 43,7 & 26,6 & 31,3 \\
\hline
\end{tabular}

HA: hipertensão arterial.

A tabela 2 apresenta as prevalências estratificadas por sexo e faixa etária. A prevalência de HA foi ligeiramente maior entre as mulheres $(33,0 \mathrm{vs}$
$28,1 \%)$, ao passo que a prevalência de obesidade foi similar entre os sexos.

Tabela 2. Prevalência de sobrepeso, obesidade e HA por sexo e faixa etária.

\begin{tabular}{lcccccc}
\hline Sexo/Faixa etária & $\mathbf{n}$ & \% Baixo Peso & \% Peso Normal & \% Sobrepeso & \% Obesidade & \% HAS \\
\hline Masculino & & & & & & \\
$20-29$ & 28 & 3,6 & 32,1 & 32,1 & 32,1 & 3,3 \\
$30-39$ & 41 & 0,0 & 26,8 & 46,3 & 26,8 & 3,3 \\
$40-49$ & 63 & 0,0 & 19,0 & 50,8 & 30,2 & 26,2 \\
$50-59$ & 44 & 0,0 & 20,5 & 52,3 & 27,3 & 36,1 \\
$\geq 60$ & 41 & 2,4 & 24,4 & 56,1 & 17,1 & 31,1 \\
Total & 217 & 0,9 & 23,5 & 48,8 & 26,7 & 28,1 \\
Feminino & & & & & & \\
$20-29$ & 62 & 3,2 & 43,5 & 27,4 & 25,8 & 2,2 \\
$30-39$ & 93 & 3,2 & 41,9 & 29,0 & 25,8 & 15,4 \\
$40-49$ & 100 & 0,0 & 28,0 & 43,0 & 29,0 & 16,2 \\
$50-59$ & 87 & 0,0 & 20,7 & 56,3 & 23,0 & 27,9 \\
$\geq 60$ & 70 & 0,0 & 24,3 & 47,1 & 28,6 & 38,2 \\
Total & 412 & 1,2 & 31,3 & 41,0 & 26,5 & 33,0 \\
\hline
\end{tabular}

HA: hipertensão arterial.

O teste Qui-Quadrado mostrou associação significativa entre HA e excesso de peso $\left(\chi^{2}=17,48\right.$; $\mathrm{p}=0,001)$, e entre HA e idade $\left(\chi^{2}=112,88 ; \mathrm{p}<0,001\right)$.

A figura 1 apresenta as prevalências de sobrepeso, obesidade, excesso de peso e HA observa- das no presente estudo, bem como as prevalências relatadas no VIGITEL ${ }^{2}$. Observa-se que as prevalências são maiores na comunidade avaliada no presente estudo quando comparadas aos dados nacionais e do DF como um todo. 
Figura 1. Comparativo das prevalências de sobrepeso, obesidade, excesso de peso e hipertensão arterial entre o VIGITEL e a comunidade avaliada.

80

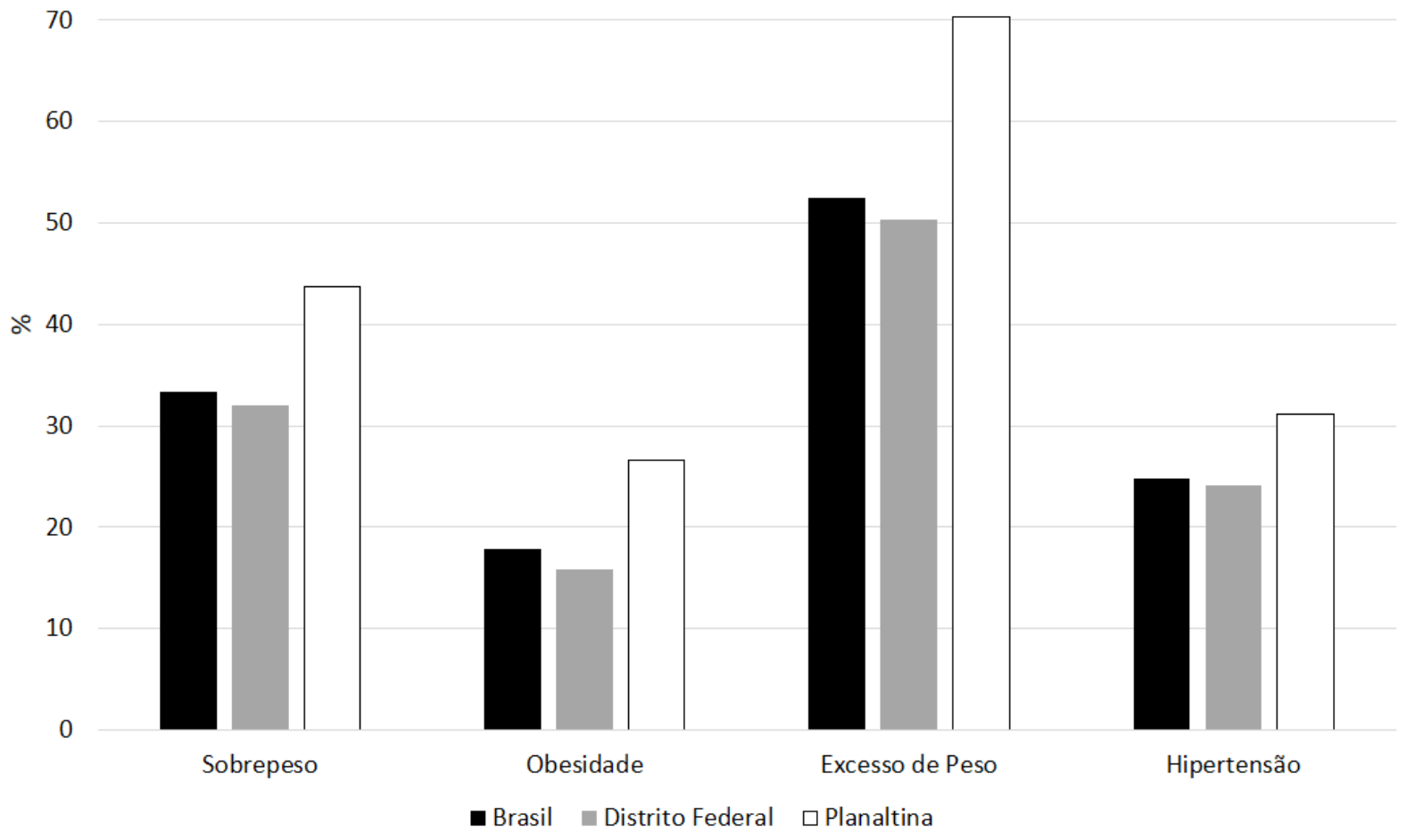

\section{Discussão}

O principal achado deste estudo é que as prevalências de HA e obesidade na comunidade avaliada são maiores do que os valores relatados em nível nacional e distrital. De acordo com o VIGI$\mathrm{TEL}^{2}$, a prevalência de HA no conjunto das capitais brasileiras e no DF é $24,8 \%$ e $24,2 \%$, respectivamente. Em Planaltina, observou-se 31,1\%.

Adicionalmente, a prevalência de $\mathrm{HA}$ em Planaltina é maior do que a encontrada em outros estudos de base populacional urbana ${ }^{5,6}$. Costa e colaboradores $^{5}$ relataram prevalência de 23,6\% em Pelotas, enquanto Feijão et al. $^{6}$ observaram HA em 22,5\% da população de Caucaia, região metropolitana de Fortaleza. Digno de nota, esses dois estudos avaliaram a prevalência por meio da medida da PA, ao passo que no presente estudo, foi utilizado o relato do participante. A prevalência de HA em Planaltina também foi maior do que a observada recentemente em outros países, como a Inglaterra (30,0\%), Estados Unidos (29,1\%) e Canadá $(19,5 \%)^{7}$.

Ademais, a presença de HA aumenta com o aumento da faixa etária em ambos os sexos. A associação entre PA e idade é conhecida ${ }^{5,8}$. Apesar disso, os idosos da comunidade estudada apresentaram prevalência um pouco menor (36\%) do que aqueles de outros países de baixa e média renda, como a China (42,7\%), Índia (37,8\%) e México (44,6\%).

A associação entre PA e excesso de peso também é conhecida ${ }^{6,9}$. Digno de destaque foi a alta prevalência de sobrepeso $(43,7 \%)$ e obesidade $(26,6 \%)$ na comunidade avaliada. Voluntários com excesso de peso (IMC $\geq 25 \mathrm{~kg} / \mathrm{m}^{2}$ ) correspondem a 70,3\% da amostra, sendo 75,5\% entre os homens e $67,5 \%$ entre as mulheres. Esses achados são preocupantes, tendo em vista que os valores são mais elevados do que os relatados pelo Ministério da Saúde $^{2}$ no Brasil (17,9\% de obesidade; $52,5 \%$ de excesso de peso) e no DF (15,8\% de obesidade; $50,3 \%$ de excesso de peso). 
Vale ressaltar que o VIGITEL é um inquérito telefônico, ao passo que a obesidade foi classificada, neste estudo, de acordo com as medidas antropométricas. Contudo, a comparação entre os dados encontrados nesse estudo com o VIGITEL é pertinente, tendo em vista que a literatura recomenda a vigilância epidemiológica de doenças crônicas por meio de inquérito telefônico ${ }^{10}$.

Outros estudos encontraram prevalência de excesso de peso de $51,2 \%$ na região metropolitana de Fortaleza $^{6}$ e 52,3\% em Belo Horizonte ${ }^{11}$. A obesidade é, de fato, um desafio à saúde pública em todo o mundo. Nos Estado Unidos, por exemplo, a prevalência dessa doença era de $34,4 \%$ em $2011^{12}$, chegando a $39,7 \%$ entre idosos ${ }^{13}$. Na presente investigação, a prevalência de obesidade foi similar entre os sexos, sendo um pouco maior nas idosas $(28,6 v s$ 17,1\%).

A prevalência de HA e obesidade têm aumentado no Brasil nos últimos anos ${ }^{14}$. Parte da explicação reside nas mudanças de estilo de vida (padrão de atividade física e alimentação) associadas ao desenvolvimento urbano e ao processo de industrialização ${ }^{8}$. Particularmente, Planaltina é uma cidade próxima a Brasília caracterizada por muitos moradores que trabalham na capital federal durante o dia e, possivelmente, adotam hábitos não saudáveis em sua rotina. Ademais, são afligidos por serviços públicos tradicionalmente insuficientes.

Os resultados ora apresentados revelam prevalência preocupante de excesso de peso e HA e apontam para a premência de ações do poder público na promoção da saúde da comunidade estudada. Digno de menção, a HA e a obesidade (particularmente a circunferência da cintura elevada) são componentes da síndrome metabólica que, por sua vez, aumenta em até três vezes o risco de desenvolvimento de doenças cardiovasculares e em cinco vezes o risco de desenvolvimento de diabetes melito do tipo $2^{15}$. Essas informações reforçam a importância de ações que objetivem a melhora do perfil antropométrico e de pressão arterial na comunidade avaliada.

O presente relato apresenta lacunas que devem ser reconhecidas e preenchidas em estudos futuros, como a avaliação de outros fatores associados ao excesso de peso e a HA, tais como as condições socioeconômicas, nível de atividade física, etnia, tabagismo e etilismo etc. Além disso, o diagnóstico de HA por meio de monitoramento ambulato- rial deve ser considerado em futuras intervenções na população estudada. Não obstante, esta investigação contribui sobremaneira com a identificação do perfil antropométrico de uma amostra significante da comunidade avaliada e aponta para a necessidade de mais investigações e ações de saúde pública no local.

\section{Conclusão}

A comunidade avaliada no presente estudo apresenta prevalência alta de obesidade e hipertensão arterial. Para além disso, tais prevalências são superiores àquelas encontradas em outras regiões do país e no próprio Distrito Federal como um todo. Com isso, ações que objetivem a melhora do perfil antropométrico e de pressão arterial são prementes na comunidade avaliada.

\section{Agradecimentos}

Os autores agradecem ao $14^{\circ}$ Batalhão da Polícia Militar - Planaltina, DF e ao senhor Silom Alves Brandão pelo apoio logístico na realização da pesquisa. Pelo apoio logístico e técnico, agradecemos ao Professor Esp. Adimário Rocha Barreto e aos técnicos do CEP-ETSP que contribuíram com a coleta de dados.

\section{Referências}

1. Sociedade Brasileira de Cardiologia / Sociedade Brasileira de Hipertensão / Sociedade Brasileira de Nefrologia. VI Diretrizes Brasileiras de Hipertensão. Arq Bras Cardiol. 2010;95(1)1-51.

2. Brasil. Ministério da Saúde. Secretaria de Vigilância em Saúde. Vigitel Brasil 2014: vigilância de fatores de risco e proteção para doenças crônicas por inquérito telefônico. Brasília, DF: Ministério da Saúde; 2015. 152 p.

3. Associação Brasileira para o Estudo da Obesidade e da Síndrome Metabólica (Abeso). Diretrizes Brasileiras de Obesidade 2009-2010. 3.ed. Itapevi, SP: AC Farmacêutica; 2009.

4. Carneiro G, Faria NA, Ribeiro Filho FF, Guimarães A, Lerário D, Ferreira SRG, et al. Influência da distribuição da gordura corporal sobre a prevalência de hipertensão arterial e outros fatores de risco cardiovascular em indivíduos obesos. Rev Assoc Med Bras. 2003;49(3):306-11. 
5. Costa JSD da, Soares J, Barcellos FC, Sclowitz ML, Krolow I, Sclowitz T, et al. Prevalência de Hipertensão Arterial em Adultos e Fatore Associados: um estudo de base populacional urbana em Pelotas, Rio Grande do Sul, Brasil. Arq Bras Cardiol. 2007;88:59-65.

6. Feijão AMM, Gadelha FV, Bezerra AA, Oliveira AM, Silva MDSS, Lima JWDO. Prevalência de excesso de peso e hipertensão arterial em população urbana de baixa renda. Arq Bras Cardiol. 2005;84(1):29-33.

7. Joffres M, Falaschetti E, Gillespie C, Robitaille C, Loustalot F, Poulter N, et al. Hypertension prevalence, awareness, tretament and control in national surveys from England, the USA and Canada, and correlation with stroke and ischaemic heart disease mortality: a cross-sectional study. BMJ Open. 2013;3(8):e003423.

8. Lloyd-Sherlock P, Beard J, Minicuci N, Ebrahim S, Chatterji S. Hypertension among older adults in low- and middle-income countries: prevalence, awareness and control. Int J Epidemiol. 2014;43(1):116-28.

9. Gus M, Moreira LB, Pimentel M, Gleisener ALM, Moraes RS, Fuchs FD. Associação entre diferentes indicadores de obesidade e prevalência de hipertensão arterial. Arq Bras Cardiol. 1998;70(2):111-14.

10. Ferreira AD, César CC, Malta DC, Souza Andrade AC, Ramos CGC, Proietti FA, et al. Validade de estimativas obtidas por inquérito telefônico: comparação entre VIGITEL 2008 e inquérito Saúde em Beagá. Rev Bras Epidemiol. 2011;14(1):16-30.

11. Andrade RG, Chaves OC, Costa DAS, Andrade ACS, Bispo S, Felicissimo MF, et al. Excesso de peso em homens e mulheres residentes em área urbana: fatores individuais e contexto socioeconômico. Cad Saúde Pública. 2015;31:111 .

12. Shields M, Carroll MD, Ogden CL. Adult obesity prevalence in Canada and the United States. NCHS Data Brief. 2011;(56)1-8.

13. Ogden CL, Carroll MD, Kit BK, Flegal KM. prevalence of Obesity in the United States. NCHS Data Brief, 2012;(82):1-8.

14. Muniz LC, Cascaes AM, Wehrmeister FC, Martínez-Mesa J, Barros AJD, Menezes AMB. Trends in self-reported arterial hypertension in Brazilian adults: an analysis of data from the Brazilian National Household Sample Survey, 1998-2008. Cad Saúde Pública. 2012;28(8):1599-607.

15. Leite EB, Anchieta VCC. Identificação de síndrome metabólica em policiais civis do Distrito Federal, Brasil. Brasília Med. 2013;50(3):186-193. 